\title{
New Physics Interpretations with GAMBIT
}

\author{
Peter Athron*t \\ Monash University \\ E-mail: peter.athron@coepp.org.au
}

I present recent results from the Global and Modular Beyond-the-Standard-Model Inference Tool (GAMBIT) collaboration. Global fits with GAMBIT have been carried out on a variety of models including supersymmetric models, scalar singlet dark matter, fermionic and vector Higgs portal dark matter and axions. Here I focus on a recent GAMBIT study interpreting collider constraints on electroweakinos (arXiv:1809.02097). We show that when the neutralinos and charginos are the only light states of the MSSM, there are scenarios which evade LHC constraints for any mass of the lightest neutralino and the lightest chargino, i.e. the profile likelihood shows no constraint in this plane when one only considers the possibility of excluding new physics. Intriguingly, in addition we also find that excesses in the data can lead to closed confidence level contours, indicating a preference for light neutralinos and charginos over the Standard Model. We find the excess has a local significance of 3.3 sigma when combining ATLAS and CMS $13 \mathrm{TeV}$ searches, but this drops to 2.9 sigma when including $8 \mathrm{TeV}$ searches as well.

European Physical Society Conference on High Energy Physics - EPS-HEP2019 -

10-17 July, 2019

Ghent, Belgium

\footnotetext{
${ }^{*}$ Speaker.

${ }^{\dagger}$ On behalf of the GAMBIT collaboration.
} 


\section{GAMBIT}

The Global and Modular Beyond the Standard Model Inference Tool (GAMBIT) is a collaboration and software tool [1, 2, 3, 4, 5, 6] for global fits of Standard Model (SM) extensions. Global fits are the best way to assess the collective impact of many experiments on physics beyond the Standard Model (BSM), and is also the best way to assess the true impact of an individual experiment. Realistic extensions of the SM tend to have large multi-dimensional parameter spaces and make predictions for a wide variety of collider and astrophysical observables. To understand the impact of searches on a BSM physics theory one should do a global fit of the new physics model. To do this one should: i) combine all experimental results using rigorous statistics to determine the composite likelihood; ii) scan over the full parameter space with an intelligent sampling algorithm and iii) finally to visualize the results one may project onto planes of interest by profiling or marginalizing the composite likelihood. Global fitting can also be used for model comparisons, to demonstrate which model is favored by the data.

GAMBIT has been used for global fits on a wide variety of models, including the scalar singlet dark matter model [7, 8], fermion and vector Higgs portal dark matter [9], axions [10], right handed neutrinos [11] and global fits of supersymmetric extensions of the SM [12, 13]. Examples of parameter estimation from recent GAMBIT papers are shown in Fig. 1, where one can also see that GAMBIT can be used to obtain both Bayesian and frequentist results. GAMBIT can also be used for model comparison, for example in Ref. [9] the posterior odds are calculated between each model and also compared to scalar singlet dark matter, showing the extent to which the data discriminated between the different models of dark matter.

Another interesting recent work has been an investigation of the impact of collider constraints on electroweakinos [14]. In this contribution to the EPS-HEP2019 proceedings we focus on this work investigating the electroweakino sector of the the minimal supersymmetric Standard Model (MSSM).

\section{Electroweakinos}

Electroweakinos are fermion superpartners of Higgs bosons and electroweak gauge bosons predicted by supersymmetric extensions of the Standard Model. These states play an important role in the naturalness of the MSSM as the mass of Higgs superpartners (Higgsinos) can give a tree level mass contribution to the Higgs vacuum expectation value (VEV) that fixes the electroweak scale. If the Higgsinos are heavier than the weak scale then there must be cancellations between this mass and some other parameters in order to predict the electroweak scale found in nature. The heavier the Higgsinos are, the greater the fine tuning needed to enforce this cancellation. Furthermore the lightest neutral electroweakino (i.e. the lightest neutralino) provides a plausible dark matter candidate.

Therefore we consider a model where the particles that may be light enough to be produced at the Large Hadron Collider (LHC) are restricted to the SM particles, including a $125 \mathrm{GeV}$ SM-like Higgs boson, the neutralinos (neutral electroweakinos) and the charginos (charged electroweakinos). The neutralinos are the mass eigenstates formed from the binos, neutral winos and neutral Higgsinos, which are, respectively, superpartners of the $U(1)_{Y}$ gauge field, the neutral $S U(2)$ gauge 

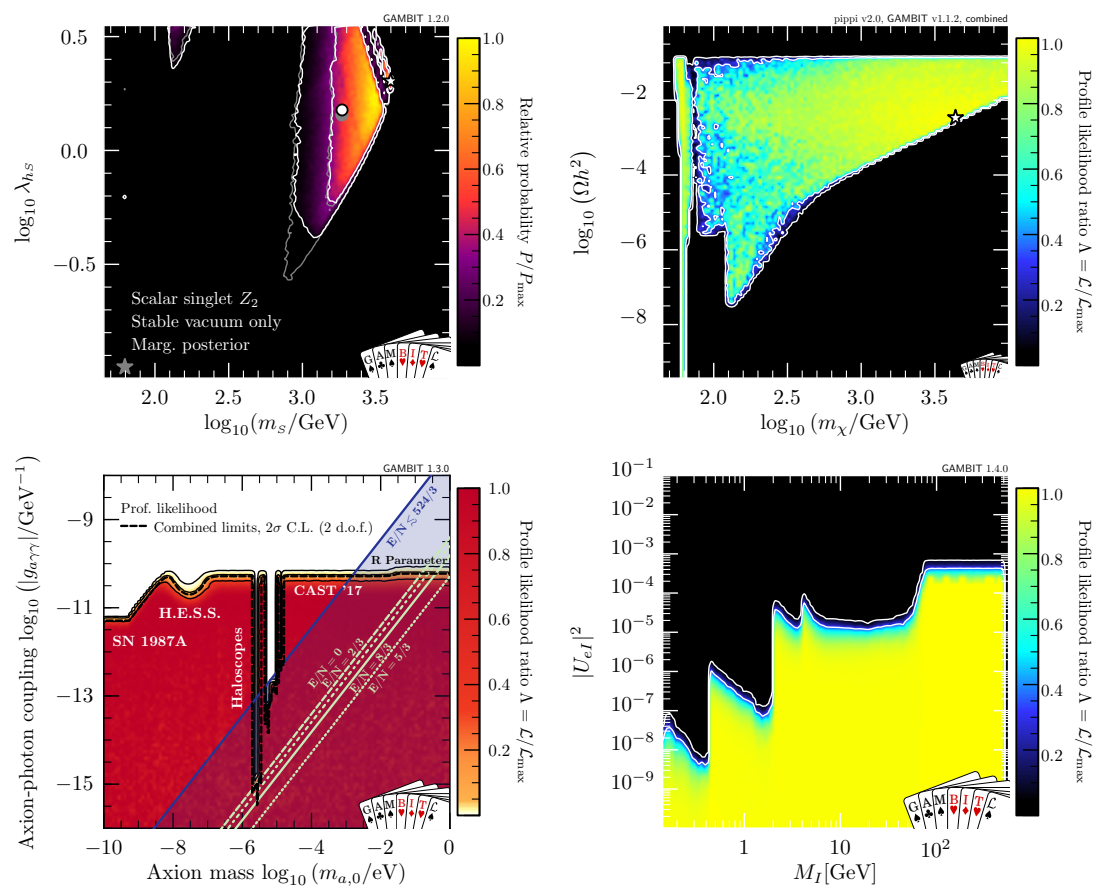

Figure 1: Results from recent papers using GAMBIT. The top left panel shows the marginalized posterior from the scalar singlet dark matter model, taken from Ref. [8]. The top right panel shows the profile likelihood for a Majorana fermion dark matter candidate taken from Ref. [9]. In the bottom left panel the profile likelihood for general axion-like particle models is shown, reproduced from Ref. [10]. In the bottom right results for the Standard Model extended by right-handed neutrinos are shown, taken from Ref. [11]. Further details can be found in the original references.

bosons, and the Higgs bosons of the MSSM. We assume all other states predicted by the MSSM are too heavy to be produced at the LHC.

The relevant parameters are the soft supersymmetry (SUSY) breaking bino and wino masses, $M_{1}$ and $M_{2}$ respectively, the Higgsino bilinear mass $\mu$ and the ratio of the electroweak VEVs $\tan \beta$. $M_{1}$ and $\mu$ are varied in the range $[-2 \mathrm{TeV},+2 \mathrm{TeV}]$, while $M_{2}$ is restricted to positive values in the range $[0,2 \mathrm{TeV}]$. The range for $\tan \beta$ is $[1,70]$.

\section{Results}

First we consider a composite likelihood computed from only $13 \mathrm{TeV}$ LHC searches for electroweakinos that were available at the time of this work and place a cap on it so that it is never greater than the SM likelihood. This is designed to test whether these searches exclude realistic electroweakinos. The complete list of searches included can be found in Table 3 of Ref. [14].

The results for this are shown in Fig. 2 in the plane of the lightest neutralino $\left(m_{\chi_{1}^{0}}\right)$ and the lightest chargino $\left(m_{\chi_{1}^{ \pm}}\right)$. Remarkably one can see that there is essentially no general constraint on any point in this plane. This result must be interpreted carefully however. This is not saying that these searches are not excluding any realistic electroweakino scenarios. In the full four dimensional parameter space many scenarios may be excluded. However these results show that there 


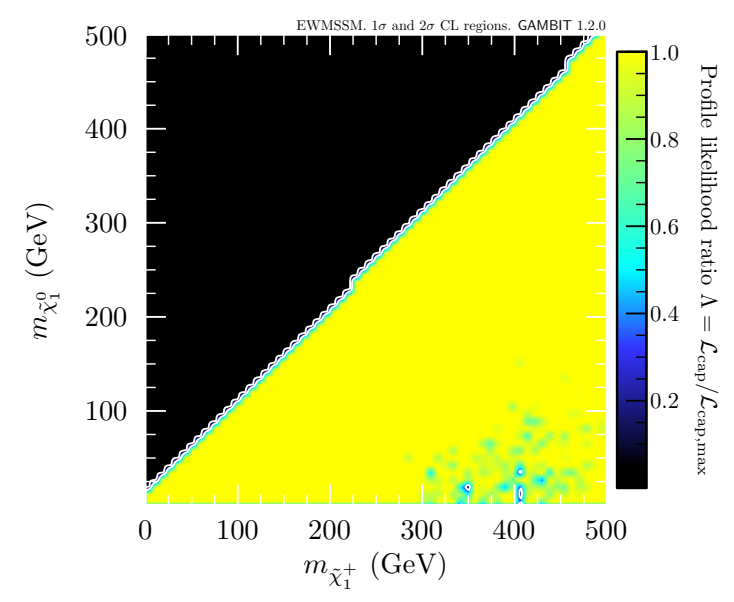

Figure 2: Capped profile likelihood from the joint likelihood for $13 \mathrm{TeV}$ LHC electroweakino searches, shown in the $m_{\chi_{1}^{0}}-m_{\chi_{1}^{ \pm}}$plane. Plot reproduced from Ref. [14].

is sufficient freedom in the MSSM electroweakino sector that for every point in the $m_{\chi_{1}^{0}}-m_{\chi_{1}^{ \pm}}$ plane there is at least one scenario on the full four dimensional parameter space that can evade the constraints from the considered $13 \mathrm{TeV}$ searches. This result says nothing about how much of the volume in the full four dimensional space is constrained however. Nonetheless it demonstrates that the simplified model interpretations of the LHC searches must not be over-interpreted as implying a general constraint on this plane. It is still possible that there are light electroweakinos that could be discovered by the LHC or a future experiment.

Next we remove the cap from the likelihood, so that any excesses in the data may also be fitted, and calculate a composite likelihood based on $13 \mathrm{TeV}$ LHC electroweakino searches, LEP electroweakino searches and Higgs and $\mathrm{Z}$ invisible widths. The results are shown in Fig. 3. Note that the in the case of a constraint one would normally expect the low mass regions to be black, i.e. "excluded" and the higher mass region to have larger profile likelihood values. Instead these results show that the data actually prefer all electroweakinos to be light, and the higher mass region (which corresponds to essentially SM-like predictions) is excluded.

As before one must be careful about the interpretation of this result and not over-interpret it. While these plots show $1 \sigma$ and $2 \sigma$ confidence limits, suggesting evidence for light electroweakinos in the data, they do not provide a clear answer as to the statistical significance of this evidence. To assess this we calculated a local p-value for the signal, where we used a Monte Carlo approach to determine the distribution of our test statistic,

$$
q_{\mathrm{LS}}=-2 \log \frac{\mathscr{L}_{\text {joint }}(\mu=1, \hat{\eta})}{\mathscr{L}_{\text {joint }}(\mu=0, \hat{\hat{\eta}})}
$$

where the numerator has the joint likelihood for analyses and the denominator has the joint likelihood for the background-only hypothesis which we construct by setting the signal to zero.

We find that the local significance is $3.3 \sigma$. This result includes only the $13 \mathrm{TeV}$ searches, however given these results it is clear that $8 \mathrm{TeV}$ results they are supposed to supersede can also have an impact. Therefore we repeated our analyses including also $8 \mathrm{TeV}$ searches and this reduced 

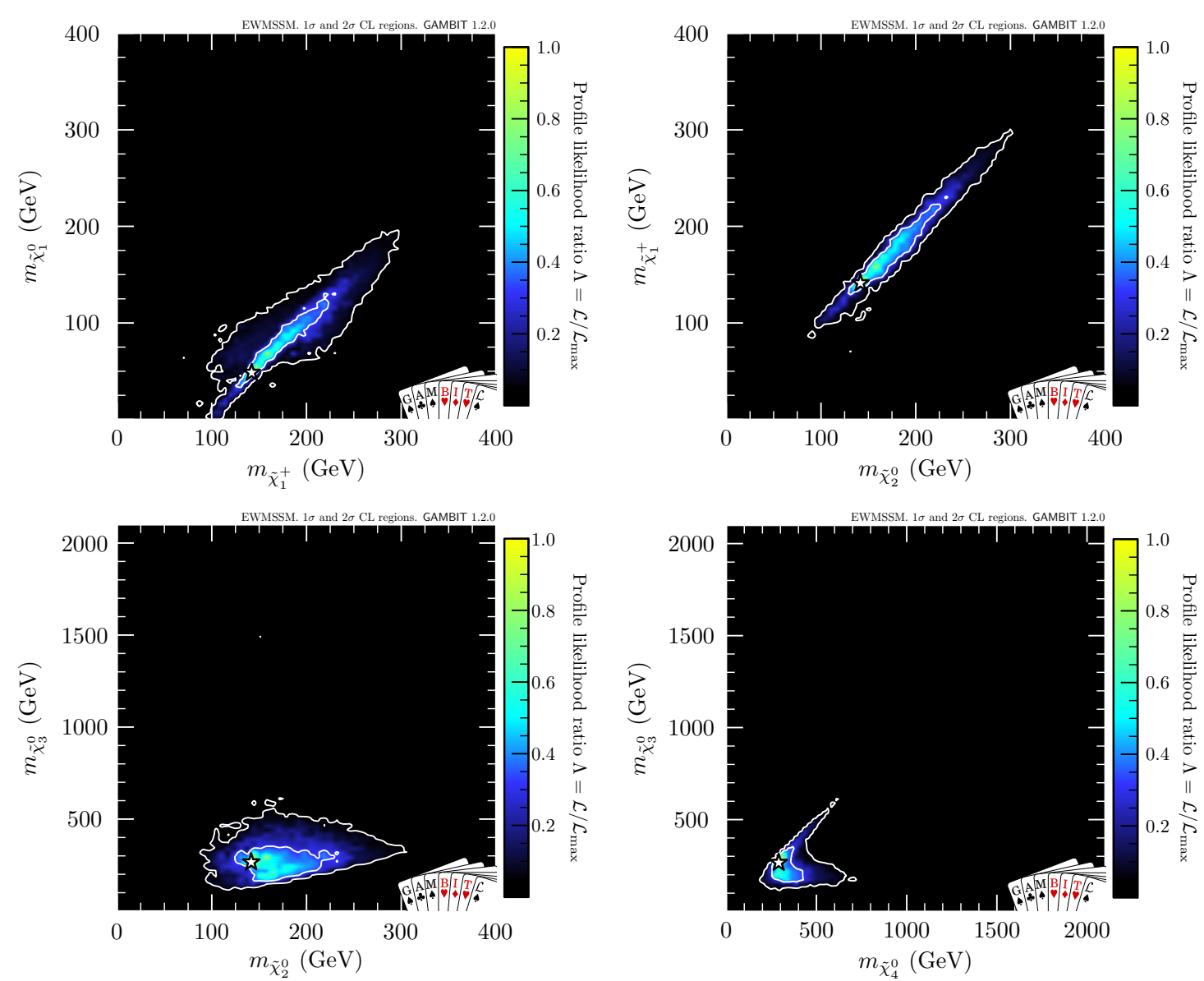

Figure 3: Profile likelihood, where the likelihood includes $13 \mathrm{TeV}$ LHC electroweakino searches, LEP electroweakino searches and Higgs and $\mathrm{Z}$ invisible widths. Results are shown in various planes of the electroweakino masses, specifically $\left(m_{\tilde{\chi}_{1}^{ \pm}}, m_{\tilde{\chi}_{1}^{0}}\right)$ (upper left), $\left(m_{\tilde{\chi}_{2}^{0}}, m_{\tilde{\chi}_{1}^{ \pm}}\right)$(upper right), $\left(m_{\tilde{\chi}_{2}^{0}}, m_{\tilde{\chi}_{3}^{0}}\right)$ (lower left) and $\left(m_{\tilde{\chi}_{4}^{0}}, m_{\tilde{\chi}_{3}^{0}}\right)$ (lower right). The $1 \sigma$ and $2 \sigma$ confidence regions are shown as contour lines and a white star is shown at the best-fit point. Plots reproduced from Ref. [14].

the local p-value to $2.9 \sigma$. A second extremely important caveat here is that this local p-value does not take account of the look else where effect. This will reduce the significance of the result. In any case the combined significance of the anomalies is intriguing but smaller than other excesses that have come and gone in recent years.

Nonetheless a very interesting feature of our results relates to dark matter. Since we wanted to focus only on the direct impact of the collider searches, unlike our previous work, we did not target explanations of dark matter in our scan by including the relic density of dark matter or non-collider dark matter searches in our likelihood. Indeed we assumed that all other SUSY particles are heavy removing the possibility of sfermion co-annihilation and heavy Higgs funnel mechanisms being present to deplete the relic density to the observed value (or below).

However despite this the excesses automatically pushed us towards a region of the parameter space where the relic density could be depleted by an SM-Higgs or Z-boson funnel mechanisms. Therefore we post-processed our results to add likelihood contributions from the relic density of 
dark matter and direct detection of dark matter. The results of this are shown in Fig. 4. Remarkably we find that some of our samples can achieve a relic density at or below the observed value and evade direct detection constraints.
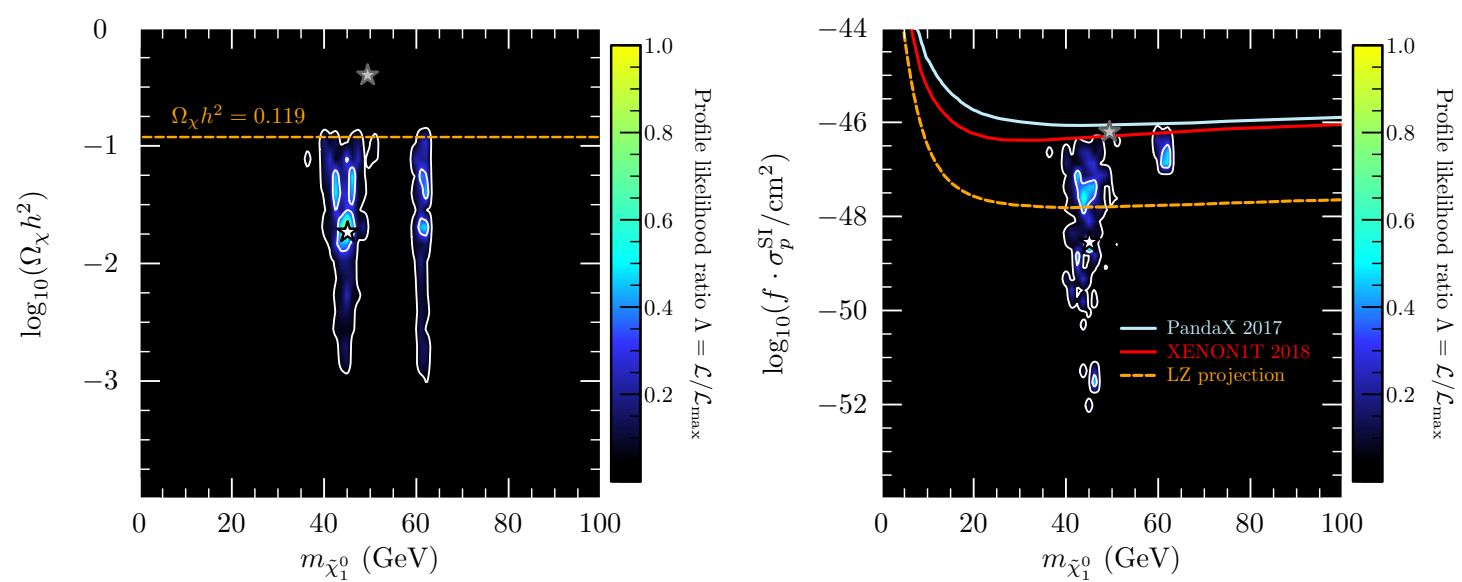

Figure 4: Profile likelihood with contributions from the LEP and LHC collider likelihoods used in the previous plot, and additional contributions from the relic density of dark matter and direct detection of dark matter. In the left panel the results are shown for the relic density against the LSP mass, while the right panel shows the results in the plane of the spin-independent direct detection cross-section and the LSP mass. As before $1 \sigma$ and $2 \sigma$ regions are shown with contour lines. A white star marks the best fit point from the full composite likelihood, while the collider-only best-fit point is indicated by a grey star. Plots reproduced from Ref. [14].

\section{Conclusions}

Our results show that there is no general exclusion on light electroweakinos from LHC. In fact there are some excesses in the data that favor certain light electroweakino scenarios. Furthermore a subset of the scenarios favored by the collider searches can explain the relic density of dark matter. These results demonstrate that future electroweakino searches at the LHC can be very interesting, but our results should also be interpreted with great care. The significance of collider excesses is intriguing but not large enough to celebrate, and could easily just be statistical fluctuations. The main lesson from this work is we must be careful how we interpret simplified model search results. Treating them as general exclusions on non-simplified models can exaggerate limits and could even miss statistically significant anomalies in the data that indicate new physics!

\section{References}

[1] P. Athron et al. [GAMBIT Collaboration], Eur. Phys. J. C 77, no. 11, 784 (2017) Addendum: [Eur. Phys. J. C 78, no. 2, 98 (2018)] doi:10.1140/epjc/s10052-017-5513-2, 10.1140/epjc/s10052-017-5321-8 [arXiv:1705.07908 [hep-ph]].

[2] C. Balázs et al. [GAMBIT Collaboration], Eur. Phys. J. C 77, no. 11, 795 (2017) doi:10.1140/epjc/s10052-017-5285-8 [arXiv:1705.07919 [hep-ph]]. 
[3] F. U. Bernlochner et al. [The GAMBIT Flavour Workgroup], Eur. Phys. J. C 77, no. 11, 786 (2017) doi:10.1140/epjc/s10052-017-5157-2 [arXiv:1705.07933 [hep-ph]].

[4] T. Bringmann et al. [The GAMBIT Dark Matter Workgroup], Eur. Phys. J. C 77, no. 12, 831 (2017) doi:10.1140/epjc/s10052-017-5155-4 [arXiv:1705.07920 [hep-ph]].

[5] P. Athron et al. [GAMBIT Models Workgroup], Eur. Phys. J. C 78, no. 1, 22 (2018) doi:10.1140/epjc/s10052-017-5390-8 [arXiv:1705.07936 [hep-ph]].

[6] G. D. Martinez et al. [GAMBIT Collaboration], Eur. Phys. J. C 77, no. 11, 761 (2017) doi:10.1140/epjc/s10052-017-5274-y [arXiv:1705.07959 [hep-ph]].

[7] P. Athron et al. [GAMBIT Collaboration], Eur. Phys. J. C 77, no. 8, 568 (2017) doi:10.1140/epjc/s10052-017-5113-1 [arXiv:1705.07931 [hep-ph]].

[8] P. Athron, J. M. Cornell, F. Kahlhoefer, J. Mckay, P. Scott and S. Wild, Eur. Phys. J. C 78, no. 10, 830 (2018) doi:10.1140/epjc/s10052-018-6314-y [arXiv:1806.11281 [hep-ph]].

[9] P. Athron et al. [GAMBIT Collaboration], Eur. Phys. J. C 79, no. 1, 38 (2019) doi:10.1140/epjc/s10052-018-6513-6 [arXiv:1808.10465 [hep-ph]].

[10] S. Hoof, F. Kahlhoefer, P. Scott, C. Weniger and M. White, JHEP 1903, 191 (2019) doi:10.1007/JHEP03(2019)191 [arXiv:1810.07192 [hep-ph]].

[11] M. Chrzaszcz, M. Drewes, T. E. Gonzalo, J. Harz, S. Krishnamurthy and C. Weniger, arXiv:1908.02302 [hep-ph].

[12] P. Athron et al. [GAMBIT Collaboration], Eur. Phys. J. C 77, no. 12, 824 (2017) doi:10.1140/epjc/s10052-017-5167-0 [arXiv:1705.07935 [hep-ph]].

[13] P. Athron et al. [GAMBIT Collaboration], Eur. Phys. J. C 77, no. 12, 879 (2017) doi:10.1140/epjc/s10052-017-5196-8 [arXiv:1705.07917 [hep-ph]].

[14] P. Athron et al. [GAMBIT Collaboration], Eur. Phys. J. C 79, no. 5, 395 (2019) doi:10.1140/epjc/s10052-019-6837-x [arXiv:1809.02097 [hep-ph]]. 